\title{
PISACov: Expanding jsPISA with evolutionary covariance data to better determine protein quaternary state from a crystal structure
}

\author{
J.J. Burgos-Mármol'1, R.M. Keegan², E. Krissinel², D.J. Rigden ${ }^{1}$ \\ ${ }^{1}$ Institute of Systems, Molecular and Integrative Biology, University of Liverpool, Liverpool L69 7ZB, UK \\ ${ }^{2}$ UKRI-STFC, Rutherford Appleton Laboratory, Research Complex at Harwell, Didcot OX11 OFA, UK \\ J.J.Burgos-Marmol@liverpool.ac.uk
}

Reliable determination of the quaternary structure of a protein is often crucial to a full understanding of its function. However, for decades, crystallographers have sometimes struggled to distinguish between biologically meaningful interfaces observed in a crystal structure, and the unnatural lattice contacts that allow for crystal formation. In order to solve this problem, Eugene Krissinel developed PISA, and later jsPISA, a CCP4 tool that sorts different candidate quaternary structures according to a likelihood obtained from results on dissociation free energy $[1,2]$. Additionally, jsPISA incorporates an "interaction radar" that allows for a rapid visualisation of how likely an interface is according to a number of physico-chemical parameters.

In order to provide jsPISA with an additional -- independent -- source of information to determine the probability of a given interface to biologically exist, we propose the use of evolutionary covariance data. This proposal is based upon the observation that pairs of residues whose interaction contributes to a biologically important interface are constrained in their evolution [3,4]. Consequently, the detection of a pair covariation signal points at the existence of a contact between the two residues contributing to the formation of a biologically important interface. The new extension, named PISACov, aims to enhance the results currently displayed by jsPISA with an additional score and new data based on evolutionary covariance analysis, thereby helping determine the relevant quaternary structure in difficult cases.

[1] Krissinel, E.; Henrick, K. J. (2007) Mol Biol., 372, 774-797.

[2] Krissinel, E. (2015) Nucleic Acids Research, 43, W314-W319.

[3] Ovchinnikov, S.; Kamisetty, H.; Baker, D. (2014) eLife, 3:e02030.

[4] Hopf, T.A. et al. (2014) eLife, 3:e03430.

Keywords: Evolutionary covariance; Contact prediction; Structural bioinformatics; Macromolecular interfaces; Quaternary structure

J.J.B.M acknowledges BBSRC Grant no BB/S007105/1. 